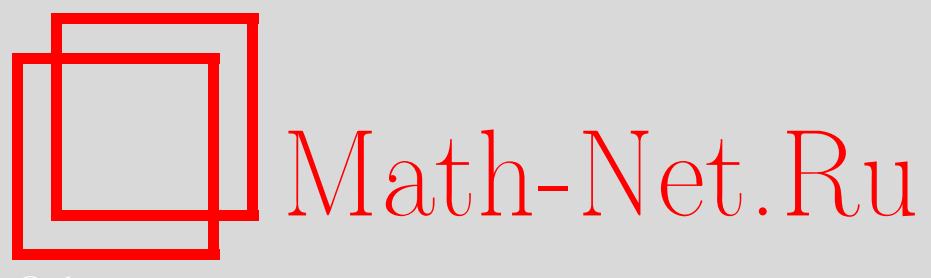

О. И. Мохов, Двойственность в специальном классе подмногообразий и фробениусовы многообразия, УМH, 2008, том 63, выпуск 2, 177-178

DOI: https://doi.org/10.4213/rm9187

Использование Общероссийского математического портала Math-Net.Ru подразумевает, что вы прочитали и согласны с пользовательским соглашением http://www . mathnet.ru/rus/agreement

Параметры загрузки:

IP : 3.89 .197 .203

26 апреля 2023 г., $17: 22: 33$

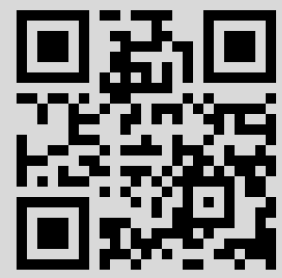




\section{Двойственность в специальном классе подмногообразий и фробениусовы многообразия}

\section{О. И. Мохов}

Рассмотрим вполне неизотропные $N$-мерные подмногообразия $M^{N}$ в $(N+L)$-мерных псевдоевклидовых пространствах $\mathbb{E}_{k}^{N+L}$ (т. е. $N$-мерные подмногообразия, которые не касаются изотропных конусов объемлющего $(N+L)$-мерного псевдоевклидова пространства ни в одной точке). Пусть $\left(z^{1}, \ldots, z^{N+L}\right)$ - псевдоевклидовы координаты в $\mathbb{E}_{k}^{N+L}$; подмногообразие $M^{N}$ задано вектор-функцией $r=\left(z^{1}\left(u^{1}, \ldots, u^{N}\right), \ldots\right.$, $\left.z^{N+L}\left(u^{1}, \ldots, u^{N}\right)\right), \operatorname{rank}\left(\partial z^{i} / \partial u^{j}\right)=N ; \partial r / \partial u^{i}=r_{i}(u), 1 \leqslant i \leqslant N$, - базис касательного пространства в произвольной точке $u=\left(u^{1}, \ldots, u^{N}\right)$ подмногообразия $M^{N}$; $n_{1}(u), \ldots, n_{L}(u)$ - произвольный базис нормального пространства $\mathbb{N}_{u}$, гладко зависящий от точки $u ; g_{i j}(u)=\left(r_{i}, r_{j}\right), 1 \leqslant i, j \leqslant N$, - первая квадратичная форма подмногообразия $\left((\cdot, \cdot)\right.$ - псевдоевклидово скалярное произведение в $\left.\mathbb{E}_{k}^{N+L}\right), h_{\alpha \beta}(u)=$ $\left(n_{\alpha}, n_{\beta}\right), \quad 1 \leqslant \alpha, \beta \leqslant L$ (для вполне неизотропных подмногообразий $\operatorname{det} g_{i j}(u) \neq 0$ и $\left.\operatorname{det} h_{\alpha \beta}(u) \neq 0\right)$. Разложения Гаусса и Вайнгартена имеют вид

$$
\frac{\partial^{2} r}{\partial u^{i} \partial u^{j}}=a_{i j}^{k}(u) \frac{\partial r}{\partial u^{k}}+b_{i j}^{\beta}(u) n_{\beta}(u), \quad \frac{\partial n_{\alpha}}{\partial u^{j}}=c_{\alpha j}^{k}(u) \frac{\partial r}{\partial u^{k}}+d_{\alpha j}^{\beta}(u) n_{\beta}(u)
$$

соответственно. Для любого подмногообразия метрика $g_{i j}(u)$, функции $h_{\alpha \beta}(u)$ и коэффициенты $a_{i j}^{k}(u), b_{i j}^{\beta}(u), c_{\alpha j}^{k}(u), d_{\alpha j}^{\beta}(u)$ связаны рядом соотношений, включающим уравнения Гаусса, Кодацци и Риччи. Отметим, что в данной работе мы рассматриваем только локальную теорию подмногообразий.

Выделим специальный класс $N$-мерных подмногообразий в $2 N$-мерных псевдоевклидовых пространствах. Наша цель - выделить случай, когда базисные векторы касательных и нормальных пространств, $r_{i}(u), 1 \leqslant i \leqslant N$, и $n_{\alpha}(u), 1 \leqslant \alpha \leqslant L$, являются равноправными и двойственными друг другу. Очевидно, что условие $L=N$ является необходимым условием такой двойственности. Кроме того, необходимым условием является условие потенциальности базиса $n_{\alpha}(u): n_{\alpha}(u)=\partial n / \partial u^{\alpha}, 1 \leqslant \alpha \leqslant N$, где $n(u)$ - некоторая вектор-функция на подмногообразии.

ОПредЕлЕниЕ 1 . Базис $n_{\alpha}(u), 1 \leqslant \alpha \leqslant N$, в нормальном пространстве $\mathbb{N}_{u}$ некоторого $N$-мерного подмногообразия в $2 N$-мерном псевдоевклидовом пространстве называется потенциальным, если на подмногообразии существует вектор-функция ( тенииал нормалей) $n(u)$ такая, что $n_{\alpha}(u)=\partial n / \partial u^{\alpha}, 1 \leqslant \alpha \leqslant N$.

Если вектор-функция $n(u)$ существует, то она порождает потенциальный базис в нормальном пространстве $\mathbb{N}_{u}$ в любой системе координат.

ОПредЕлЕниЕ $2 . \quad N$-мерное подмногообразие в $2 N$-мерном псевдоевклидовом пространстве называется подмногообразием с потенииальным нормальным базисом (или подмногообразием с потенииалом нормалей), если на подмногообразии существует вектор-функция (потенциал нормалей) $n(u)$ такая, что для любой точки $u$ на подмногообразии векторы $n_{i}(u)=\partial n / \partial u^{i}, 1 \leqslant i \leqslant N$, образуют базис в нормальном пространстве $\mathbb{N}_{u}$.

Определение 2 инвариантно (не зависит от локальной системы координат).

Коэффициенты $a_{i j}^{k}(u)$ в разложении Гаусса (1) всегда являются коэффициентами связности Леви-Чивиты метрики $g_{i j}(u)$. Для подмногообразий, оснащенных потенциальным нормальным базисом, аналогичное утверждение верно и для коэффициентов $d_{\alpha j}^{\beta}(u)$ в разложении Вайнгартена (1). Рассмотрим соответствующие разложения

Работа выполнена при поддержке РФФИ (грант № 08-01-00054) и программы "Ведущие научные школы" (грант НШ-4182.2006.1). 
Гаусса и Вайнгартена:

$$
\frac{\partial^{2} r}{\partial u^{i} \partial u^{j}}=a_{i j}^{k}(u) \frac{\partial r}{\partial u^{k}}+b_{i j}^{k}(u) \frac{\partial n}{\partial u^{k}}, \quad \frac{\partial^{2} n}{\partial u^{i} \partial u^{j}}=c_{i j}^{k}(u) \frac{\partial r}{\partial u^{k}}+d_{i j}^{k}(u) \frac{\partial n}{\partial u^{k}} .
$$

Теорема 1. Функиии $h_{i j}(u)=\left(\partial n / \partial u^{i}, \partial n / \partial u^{j}\right)$ определяют ковариантную метрику на подмногообразии, а коэффициенты $d_{i j}^{k}(u)$ в разложении Вайнгартена (2) являются коэффициентами симметричной аффинной связности, согласованной с метрикой $h_{i j}(u)$, т.е. коэффициентами связности Леви-Чивиты метрики $h_{i j}(u)$. Коэффициенты $b_{i j}^{k}(u)$ и $c_{i j}^{k}(u)$ сутъ тензоры типа $(1,2)$ на подмногообразии $M^{N}$.

Теорема 2 (принцип двойственности). Для любого подмногообразия, оснащенного потенциалом нормалей $n(u)$ и задаваемого вектор-функциями $(r(u), n(u))$, вектор-функиии $(n(u), r(u))$ также задают подмногообразие, оснащенное потенииалом нормалей, для которого $n_{i}(u)$ - касательные векторы, а $r_{i}(u)$ - базисные нормальные векторы подмногообразия, при этом все объекты локальной теории таких подмногообразий двойственны друг другу, в частности, разложение Гаусса переходит в разложение Вайнгартена, а разложение Вайнгартена переходит в разложение Гаусса, уравнения Гаусса переходят в уравнения Риччи, а уравнения Риччи переходят в уравнения Гаусса, уравнения Кодации переходят в себя, метрика $g_{i j}(u)$ переходит в метрику $h_{i j}(u)$ и наоборот.

Подмногообразия с потенциалом нормалей образуют важный и богатый класс подмногообразий. Тривиальным примером таких подмногообразий являются произвольные одномерные подмногообразия псевдоевклидовых плоскостей. Общая теория подмногообразий с потенциалом нормалей, принцип двойственности для них и важные примеры будут представлены в другой статье. Частным случаем подмногообразий с потенциалом нормалей являются подмногообразия с естественными фробениусовыми структурами, построенные автором в [1]-[4] и реализующие произвольные фробениусовы многообразия (теория фробениусовых многообразий построена в [5]). В [1] и [2] автором доказано, что любое фробениусово многообразие реализуется как некоторое плоское подмногообразие с потенциалом нормалей, для которого $g_{i j}(u)=c h_{i j}(u), c=$ const $\neq 0$, где $c$ - параметр деформации, сохраняющей фробениусову структуру. Пусть $h_{i j}(u)=\eta_{i j}, \eta_{i j}=\eta_{j i}$, det $\eta_{i j} \neq 0, \eta_{i j}=$ const, $g_{i j}=c \eta_{i j}$, $c=$ const $\neq 0$. В этом случае для подмногообразий с потенциалом нормалей выполняются соотношения $a_{i j}^{k}(u)=d_{i j}^{k}(u)=0$, а также существует функция $\Phi(u)$ такая, что $b_{i j}^{k}(u)=\eta^{k s} \partial^{3} \Phi / \partial u^{s} \partial u^{i} \partial u^{j}, c_{i j}^{k}(u)=-(1 / c) b_{i j}^{k}(u), \eta^{i s} \eta_{s j}=\delta_{j}^{i}$, причем все соотношения локальной теории подмногообразий выполняются тогда и только тогда, когда функция $\Phi(u)$ удовлетворяет уравнениям ассоциативности двумерных топологических квантовых теорий поля (уравнениям Виттена-Дейкграфа-Верлинде-Верлинде, см. [5])

$$
\begin{gathered}
\frac{\partial^{3} \Phi}{\partial u^{i} \partial u^{j} \partial u^{s}} \eta^{s p} \frac{\partial^{3} \Phi}{\partial u^{p} \partial u^{k} \partial u^{l}}=\frac{\partial^{3} \Phi}{\partial u^{i} \partial u^{k} \partial u^{s}} \eta^{s p} \frac{\partial^{3} \Phi}{\partial u^{p} \partial u^{j} \partial u^{l}} . \\
\text { Список литературы }
\end{gathered}
$$

[1] O. I. Mokhov, arXiv: abs/0710.5860, 2007. [2] O. I. Mokhov, TM [3] О. И. Мохов, УМН, 59:1 (2004), 187-188. [4] О. И. Мохов, Функи. анализ и его прил., 40:1 (2006), 14-29. [5] B. Dubrovin, Lecture Notes in Math., 1620, Springer-Verlag, Berlin, 1996, $120-348$.

О. И. Мохов (O.I. Mokhov)

Московский государственный университет им. М. В. Ломоносова

E-mail: mokhov@mi.ras.ru; mokhov@bk.ru
Представлено С. П. Новиковым Принято редколлегией 03.02 .2008 